\title{
Paramagnetic Manganese in the Atherosclerotic Plaque of Carotid Arteries
}

\author{
Yury Chelyshev, ${ }^{1,2}$ Marat Gafurov, ${ }^{1}$ Igor Ignatyev, ${ }^{3}$ Alexey Zanochkin, ${ }^{3}$ \\ Georgy Mamin, ${ }^{1}$ Boris Sorokin, ${ }^{1}$ Alexandra Sorokina, ${ }^{1}$ Natalya Lyapkalo, ${ }^{1}$ \\ Nazima Gizatullina, ${ }^{3}$ Yana Mukhamedshina, ${ }^{1}$ and Sergei Orlinskii ${ }^{1}$ \\ ${ }^{1}$ Kazan Federal University, Kazan, Russia \\ ${ }^{2}$ Kazan Medical University, Kazan, Russia \\ ${ }^{3}$ Interregional Clinical Diagnostic Centre (ICDC), Kazan, Russia
}

Correspondence should be addressed to Marat Gafurov; marat.gafurov@kpfu.ru

Received 4 July 2016; Revised 9 November 2016; Accepted 20 November 2016

Academic Editor: Hanrui Zhang

Copyright (c) 2016 Yury Chelyshev et al. This is an open access article distributed under the Creative Commons Attribution License, which permits unrestricted use, distribution, and reproduction in any medium, provided the original work is properly cited.

\begin{abstract}
The search for adequate markers of atherosclerotic plaque (AP) instability in the context of assessment of the ischemic stroke risk in patients with atherosclerosis of the carotid arteries as well as for solid physical and chemical factors that are connected with the AP stability is extremely important. We investigate the inner lining of the carotid artery specimens from the male patients with atherosclerosis (27 patients, 42-64 years old) obtained during carotid endarterectomy by using different analytical tools including ultrasound angiography, X-ray analysis, immunological, histochemical analyses, and high-field (3.4 T) pulse electron paramagnetic resonance (EPR) at $94 \mathrm{GHz}$. No correlation between the stable and unstable APs in the sense of the calcification is revealed. In all of the investigated samples, the EPR spectra of manganese, namely, $\mathrm{Mn}^{2+}$ ions, are registered. Spectral and relaxation characteristics of $\mathrm{Mn}^{2+}$ ions are close to those obtained for the synthetic (nano) hydroxyapatite species but differ from each other for stable and unstable APs. This demonstrates that AP stability could be specified by the molecular organization of their hydroxyapatite components. The origin of the obtained differences and the possibility of using EPR of $\mathrm{Mn}^{2+}$ as an AP stability marker are discussed.
\end{abstract}

\section{Introduction}

Atherosclerosis is a cardiovascular disease characterized by the accumulation of fatty deposits within the arterial intima, which can eventually expand into the lumen and obstruct blood flow. Atherosclerosis is the result of chronic inflammation in the arterial vessels, initiated by endothelial cells damage, involving monocytes, macrophages, and dendritic cells in conditions of oxidative stress. It is a slowly progressive multifactorial disease with a long asymptomatic phase [1]. Aortic arch atherosclerosis (especially in the common carotid artery bifurcation) is the cause of $20 \%$ of ischemic stroke [2, $3]$, which is the consequence of the increasing atherosclerotic plaque (AP) instability.

Plaque stability becomes critical as atherosclerosis progresses. Rupture of unstable plaque can initiate thrombosis leading to vascular occlusion and downstream tissue infarction. One of the important components in the pathogenesis of atherosclerosis is an AP calcification [4,5]. The origin and mechanisms of the AP calcification remain unclear. Calcification initially affects the lipid core in the close vicinity to the cells involved in the inflammation [4]. Apoptotic bodies and cell debris act as nucleation centers for the accumulation of lipoproteins and phospholipids [3] with the deposition of biominerals, consisting mainly of the nonstoichiometric, calcium-deficient carbonated hydroxyapatite (HAp) with the chemical formulae $\mathrm{Ca}_{10}\left(\mathrm{PO}_{4}\right)_{6}\left(\mathrm{CO}_{3}\right)_{2}$ for "ideal" HAp [4-7].

At present, there is no consensus in the expert community on whether the calcification is a sign (indicator) of the AP stability. It is logical to assume that the status of the AP organomineral matrix should (or may) mirror the status of the AP stability but different aspects of calcifications must be taken into account to establish a significant relationship with the AP progress. These include chemical diversity, morphologic features at the mesoscopic and macroscopic scale, location, presence of trace elements, presence of molecular 
groups, such as carbonate groups in apatites $[6,8]$. It, in its turn, implies the comprehensive analysis of the clinical and reference materials by the variety of the analytical methods, some of which are still not widely implemented not only into the clinical practice but even into the advanced biochemical research.

Because HAp is a highly absorptive material [9-11], it is believed that this biomineral in AP would actively absorb metal complexes located in the tissue matrix as the result of release from normal or destructive cells of the vessel wall. Among those are the complexes of $\mathrm{Fe}, \mathrm{Cu}$, and $\mathrm{Mn}$, many of which are paramagnetic and, therefore, can be detected and investigated by electron paramagnetic (spin) resonance (EPR or ESR) methods (see [12, 13] and literature cited there). The type of the observed EPR spectra, their intensity, relaxation characteristics, and so forth reflect the structure and properties of the host matrix; that is, the observed paramagnetic complexes can serve as intrinsic probes that mirror the origin, growth, and status of the AP biominerals as well as a status of the adjacent tissues.

Concerning manganese, in the human body, Mn exists primarily in two oxidized states, that is, $\mathrm{Mn}^{2+}$ and $\mathrm{Mn}^{3+}$. While $\mathrm{Mn}^{3+}$ species are generally EPR silent, $\mathrm{Mn}^{2+}$ complexes are EPR detectable [14]. The most familiar manganesecontaining complex is a manganese superoxide dismutase (Mn-SOD). It is a homotetramer $(96 \mathrm{KDa})$ containing one manganese atom per subunit that cycles from $\mathrm{Mn}^{3+}$ to $\mathrm{Mn}^{2+}$ and back to $\mathrm{Mn}^{3+}$ during the two-step dismutation of superoxide. It is the primary enzyme of the antioxidant defense system that protects the energy-generating mitochondria from oxidative damage [15]. This mitochondrial enzyme catalyzes the dismutation of superoxide anions $\left(\mathrm{O}_{2} \bullet-\right)$ to hydrogen peroxide $\left(\mathrm{H}_{2} \mathrm{O}_{2}\right)$ and $\mathrm{O}_{2}$. Mn-SOD slows AP ulceration, reduces oxidized low-density lipoprotein (ox-LDL) mediated macrophage apoptosis, and inhibits endothelial cell dysfunction and the oxidation of low-density lipoproteins (LDL) by endothelial cells. Emerging evidence indicates that many cardiovascular syndromes are associated with some evidence for mitochondrial dysfunction $[1,16]$. As a result of the cell death during atherogenesis, Mn from mitochondrial Mn-SOD may appear in the extracellular matrix and could be absorbed by HAp. Whether the EPR properties of $\mathrm{Mn}^{2+}$ ions can be used in the context of the evaluation of the AP stability is an open question.

In a series of our previous papers [17-21], we mainly focused on the investigation of the natural and synthetic nano- and micropowders of HAp with the substitution by different ions and groups (lead, manganese, carbonates, nitrogen containing species) in the large concentration range of $0.001-14.0 \mathrm{wt} \%$ as well as on the comparison of the results acquired on the model systems with those obtained from the postmortem clinical material $[6,7]$. We have gathered the experience of the characterization of HAp and AP samples by different EPR techniques to choose the more sensitive high-field (HF) EPR (W-band, magnetic field $B_{0}$ of about $3.4 \mathrm{~T}$ ) which requires the substance grain size with the cross-sectional diameter of less than $1 \mathrm{~mm}$. We have noticed [17] that the relaxation characteristics, namely, the transverse/phase-memory relaxation time $T_{2 \mathrm{e}}$ of $\mathrm{Mn}^{2+}$ ions in HAp, are very sensitive to the changes in their local environment even at very low manganese concentrations of $c<0.05 \%$. On that basis, it was assumed that not only the EPR spectra but also $T_{2 \mathrm{e}}$ could correlate with the status of the AP organomineral matrix. Therefore, the HF EPR was conducted in the pulsed regime. Furthermore, by choosing the proper pulse and delay durations, one has the additional potential ability to separate the spectrally overlapped paramagnetic centres.

The main aim of this work is the experimental study of the carotid artery specimens obtained during the surgery by the pulsed HF EPR techniques [22, 23] and comparison of the results with those acquired by the established clinical, biochemical, and analytical tools.

\section{Materials and Methods}

The object of the study was the inner lining of the carotid artery specimens obtained during carotid endarterectomy in patients with atherosclerosis. We investigated 27 endarterectomy specimens (plaques or fragments of plaques) derived from 27 male patients with a mean age of 57 years (42 to 64) of whom 19 were symptomatic. All patients presented a carotid stenosis more than 60\% (North American Symptomatic Carotid Endarterectomy Trial [NASCET] criteria) as established by ultrasound (Vivid 7 Expert, GE, USA) and magnetic resonance imaging (Signa Horizon HDxt 1.5 T, GE, USA) angiography. 18 patients are smokers. Based on the modified Gray-Weale classification of atherosclerotic plaques [24, 25], 21 plaques were classified as "unstable" (echolucent, low median gray level, GSM $\leq 25$ ) while 6 as "stable" (echogenic, GSM > 25).

Sections of AP were cut and stained by hematoxylin and eosin to estimate the areas of calcium deposits from the digitized images with the help of $\times 100$ lens of Axio Imager A1 (Carl Zeiss), AxioVision version 4.8.2 (Carl Zeiss MicroImaging $\mathrm{GmbH}$ ) and ImageScope version 12.2.2.5015 (Aperio Technologies, Inc.). Shapiro-Wilk normality test, Wald-Wolfowitz runs test, and Mann-Whitney $U$ test were carried out by using StatSoft Statistica version 10.0. and Origin Pro 8.0 software products. The results were considered statistically significant at $p<0.05$.

For histology and immunohistochemistry, the samples after resection were immediately transferred into the $4 \%$ paraformaldehyde (from 95\% powder from Sigma-Aldrich, CAS number 30525-89-4). After fixation, samples were placed in $30 \%$ sucrose and a week later embedded in tissue freezing medium. Twenty microns tissue sections (fibrous caps and plaque shoulders) obtained with a Microm HM 560 Cryostat were incubated with primary and secondary antibodies. For cell characterization, samples were treated with antibodies against markers of macrophages/monocytes (CD68 antibody, Santa Cruz Biotechnology) and endothelial cells (anti-CD31, Santa Cruz Biotechnology, and anti-von Willebrand factor, anti-VWF, also known as factor VIII, Dako). Following primary antibody incubation, the reactions were visualized using Polymer Kit (Post Primary Block + Polymer, Novocastra Novolink) and AEC Peroxidase (HRP) 
Substrate Kit, 3-amino-9-ethylcarbazole (Vector Laboratories) according to the manufacturer's instructions. The numbers of CD68, CD31, and vWF immunopositive cells were quantified in the fibrous cap and shoulder region of AP in images captured using a $\times 40$ objective from five adjacent optical slices (the observed area is of $0.25 \mathrm{~mm}^{2}$ ). ImageJ version 1.46 was used for data acquisition and analysis. Data are presented as means \pm standard error of the mean (SEM). A one-way analysis of variance (ANOVA) with Tukey's test was used for comparisons between groups. Data were analyzed using Origin 8.0 software.

For EPR studies, the tissue specimens were transferred and kept in liquid nitrogen $(T=77 \mathrm{~K})$ and then freeze-dried (lyophilized) at $P=5 \cdot 10^{-5} \mathrm{mBar}$ and $T=253 \mathrm{~K}\left(-20^{\circ} \mathrm{C}\right)$. Bruker Elexsys 580/680 spectrometer operating at W-band (microwave frequency of about $93.5 \mathrm{GHz}$ ) equipped with the superconducting magnetic system and the liquid helium temperature controller was used in the pulsed regime. In this research, we have investigated the plaque shoulders and cores. Electron spin echo (ESE) EPR spectra were recorded by means of field-swept two-pulse echo sequence $\pi / 2-\tau-\pi$ with the pulse length of $\pi$ pulse of $12 \mathrm{~ns}$ and time delay $\tau=240 \mathrm{~ns}$. For the phase-memory $T_{2 \mathrm{e}}$ time measurements $\tau$ was varied from 200 ns up to the desired value with the minimal possible step of $4 \mathrm{~ns}$. The EPR measurements were conducted in the temperature range of $8-50 \mathrm{~K}$. At higher temperatures, fast spin-lattice relaxation of $\mathrm{Mn}^{2+}$ ions prevents the observation of ESE. More details concerning the different aspects of EPR applications and instrumentation for the biological related research can be found in $[13,22,23]$ and literature cited there.

All participants expressed their prior written consent to take part in the research. The study was approved by the Bioethical Committee at the ICDC Clinical Centre and was carried under the guidance of the Declaration of Helsinki (1964 and later versions).

Pure and manganese-containing synthetic hydroxyapatite nano- and micropowders with the crystallite sizes from $20 \mathrm{~nm}$ up to $1000 \mathrm{~nm}$ were synthesized by the wetprecipitation technique in the group of V.I. Putlyaev (M.V. Lomonosov Moscow State University, Moscow, Russia). Details of the synthesis and HAp characterization are given in $[17,20,21]$.

Lyophilized powder of Mn-SOD from E. coli was purchased from Sigma-Aldrich (Product number S5639-3KU) and was used as it is to take the EPR spectra.

$\mathrm{X}$-ray diffractometry (XRD) measurements were done on Bruker D8 Advance (Cu anode, $1.6 \mathrm{~kW}$, Si sample holder for the small substance amount) and analyzed in EVA program.

\section{Results}

XRD analysis (Figure 1) shows the presence of the crystal phase of hydroxyapatite in all the studied AP specimens (ID = 1011242 from the crystallography open database COD [26]). The broadenings of the peaks are mainly due to the small crystallite sizes of HAp in APs (less than $100 \mathrm{~nm}$ ). The crystallinity degree was estimated from the broad halo and varies in the range of $(40 \pm 18) \%$. No differences in the

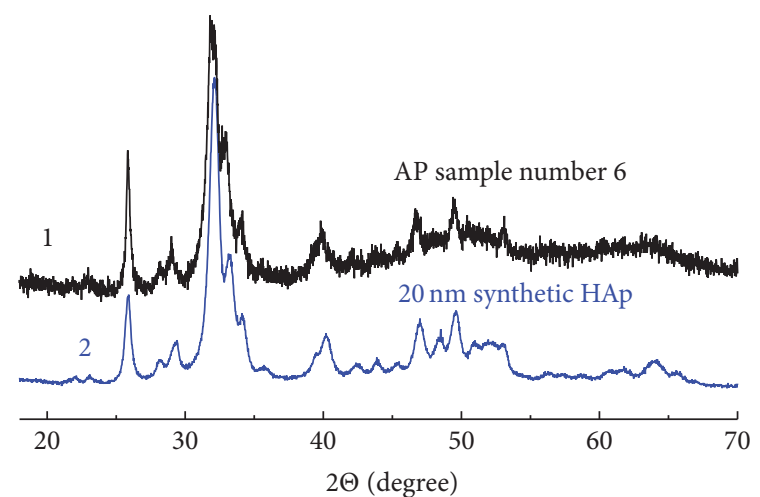

FIGURE 1: Comparison of XRD patterns for AP sample number 6 (curve 1) and the nanosized synthetic HAp (curve 2) taken in the same experimental conditions.

positions of the peaks in the XRD pattern for the investigated specimens ( 3 stable and 3 unstable plaques) were found.

The intensity (the ratio signal-to-noise) of XRD patterns varies significantly (two orders of magnitude) with sample but the quantitative estimation of amount of HAp in the investigated species is a quite tricky task and is not done in the present research.

Table 1 demonstrates that one cannot distinguish stable AP from the unstable one from the area occupied by calcium deposits. No statistically reliable differences were found for number of macrophages (CD $68^{+}$cells) for stable and unstable plaques. It is in accordance with the results of Pelisek et al. [27], for example. The difference between the stable and unstable plaques was established by the criterion of neovascularization. Thus, the number of endothelial cells counted by help of the markers CD31 and vWF in unstable plaques is higher than in the stable plaques. These data are also consistent with the results of Pelisek et al. [27].

In all of the obtained species, an EPR signal due to the presence of $\mathrm{Mn}^{2+}$ ions was observed (Figure 2). The integral intensity (and, therefore, the concentration of the $\mathrm{Mn}^{2+}$ ions) varies from sample to sample in the range of about one order of magnitude. The absolute maximal concentration of paramagnetic centers was estimated to be less than $10^{14}$ paramagnetic spins per gram of tissue under investigation. No relationship between the intensity and AP stability was observed. Because as it was pointed out above the determination of amount of inorganic phase in the investigated species was problematic, we cannot compare the concentrations of $\mathrm{Mn}^{2+}$ in different samples directly, from the comparison of the integral intensities of their EPR spectra.

Figure 2 presents the typical EPR spectra of stable (number 23) and unstable (number 27) AP samples in comparison with the manganese-containing nano-HAp and Mn-SOD. Due to the presence of the nuclear magnetic moment $I=$ $5 / 2$ for ${ }^{55} \mathrm{Mn}$, the hyperfine interaction between the electron $(S=5 / 2)$ and nuclear spins leads to the observation of the sixth-line pattern centered at $g$-factor of $g \approx 2.001(1)$ and hyperfine constant $A$ of about (9-10) mT. Closeness of $g$ and $A$ parameters to those obtained in the manganese-containing 
TABLE 1: Patients information, the results of microscopy for determination of calcium depositions $\left(\mathrm{mm}^{2}\right)$, frequency of anti-inflammatory cells in unstable and stable plaques (cells $/ 0.25 \mathrm{~mm}^{2}$ ), and EPR transverse electronic relaxation times ( $\mu$ s) for stable and unstable AP (mean \pm SEM). The number of specimens is denoted as $n$.

\begin{tabular}{lccc}
\hline Plaque feature & Stable & Unstable & $p$ value \\
\hline Patient information & $n=6$ & $n=21$ & $56.6 \pm 6.0$ \\
Average age & $57.3 \pm 6.9$ & 14 & $n=10$ \\
Smokers & 4 & $0.611 \pm 0.526$ & 0.26 \\
Calcium deposition square & $n=6$ & & Mann-Whitney U test \\
& $0.647 \pm 0.637$ & & 0.82
\end{tabular}

\begin{tabular}{lccc} 
& & Wald-Wolfowitz runs test \\
\hline Frequency of anti-inflammatory cells & $n=4$ & $n=14$ & $>0.05$ \\
$\mathrm{CD}^{+} 8^{+}$cells in fibrous cap & $42 \pm 4.5$ & $51.28 \pm 12.5$ & $>0.05$ \\
$\mathrm{CD}^{+}$cells in shoulder & $44.5 \pm 18.37$ & $31.46 \pm 17.7$ & $<0.01$ \\
$\mathrm{CD}^{+}$cells in fibrous cap & $14.2 \pm 4.15$ & $46.8 \pm 9.8$ & $<0.05$ \\
$\mathrm{CD}^{+}$cells in shoulder & $12.2 \pm 3.37$ & $45.7 \pm 15.5$ & $<0.05$ \\
$\mathrm{vWF}^{+}$cells in fibrous cap & $24.5 \pm 3.1$ & $43.7 \pm 7.2$ & $>0.05$ \\
$\mathrm{vWF}^{+}$cells in shoulder & $18.25 \pm 3.3$ & $27.86 \pm 11.31$ & $<$ \\
EPR relaxation data for T $\mathrm{N}$ K & $n=6$ & $n=21$ & $>0.01$ \\
$T_{\text {2efast }}$ & $0.52 \pm 0.15$ & $0.54 \pm 0.12$ & $>0.05$ \\
$T_{\text {2eslow }}$ & $3.91 \pm 0.54$ & $1.25 \pm 0.27$ & \\
\hline
\end{tabular}

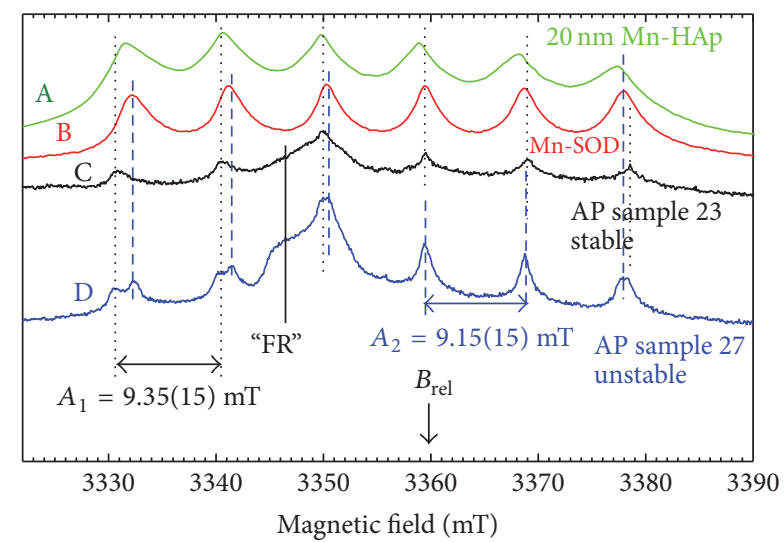

FIGURE 2: Comparison of the ESE detected EPR spectra of $20 \mathrm{~nm}$ Mn-HAp (A, green curve); Mn-SOD (B, red curve); stable AP, sample number 23, (C, black curve); unstable AP number 27 (D, blue curve). The spectra are detected at $T=50 \mathrm{~K}$. The dotted and dashed lines indicate the hyperfine components of two presumably differently located manganese ions in the crystal structure of HAp in the stable and unstable APs. The arrow shows the value of the magnetic field $B_{\text {rel }}$ in which the $T_{2 \mathrm{e}}$ curves (ESE decays) were measured. Solid line indicates the presence of additional paramagnetic species (mainly, carbon-centered "free" radicals, FR [6]).

HAp (see also [17] and references therein) allows for assuming that the obtained AP EPR spectra are mainly indebted to $\mathrm{Mn}^{2+}$ ions in HAp.

There are two types of $\mathrm{Mn}^{2+}$ signals that are detectable in the investigated AP samples. We found that 5 of 6 stable plaques reveal the EPR spectra which can be characterized by only single hyperfine constant $A_{1}=(9.35 \pm 0.15) \mathrm{mT}$ with $g_{1} \approx$ 2.001(1) (curve $C$ in Figure 1), while for all unstable plaques and one stable an additional constituent with $A_{2}=(9.15 \pm$ $0.15) \mathrm{mT}$ and $g_{2} \approx g_{1}-0.0005$ (1) should be taken into account (curve D in Figure 1) (due to the accuracy of our experimental setup and measurements, we are sure only in the difference between the $g$-factors of the two substituents but not in their absolute values). As it would be shown in Discussion, we connect this fact with the two different probable locations of $\mathrm{Mn}^{2+}$ in the crystal structure of HAp.

Additional line(s) observed in all 27 AP species belongs in our opinion to the different carbon-centered stable "free" radicals in the carbonated HAp (FR, cf. Figure 1). Their possible origin, correlation between the degree of calcification, spectral and relaxation parameters of FR, and the radiationinduced FR are discussed in [6] and are not in the focus of the present research.

Though the investigated samples are inhomogeneous, the presence of manganese allows for probing the local $\mathrm{Mn}^{2+}$ surrounding by the relaxation measurements. The experiment was done in the magnetic field $B_{\text {rel }}$ which corresponds to the 4 th component of the $\mathrm{Mn}^{2+}$ hyperfine pattern (see Figure 1). At $B_{\text {rel }}$, the FR and manganese EPR curves are spectrally resolved (that allows for avoiding their mutual influence) while coinciding for the two types of $\mathrm{Mn}^{2+} \mathrm{EPR}$ signals. The difference in $T_{2 \mathrm{e}}$ curves became obvious at $T<15 \mathrm{~K}$.

The dependence of the kinetics of electron spin echo amplitude decay on increase of delay time between the microwave pulses is presented in Figure 3. Each of the curves can be approximated by two exponents denoted as "fast" and 


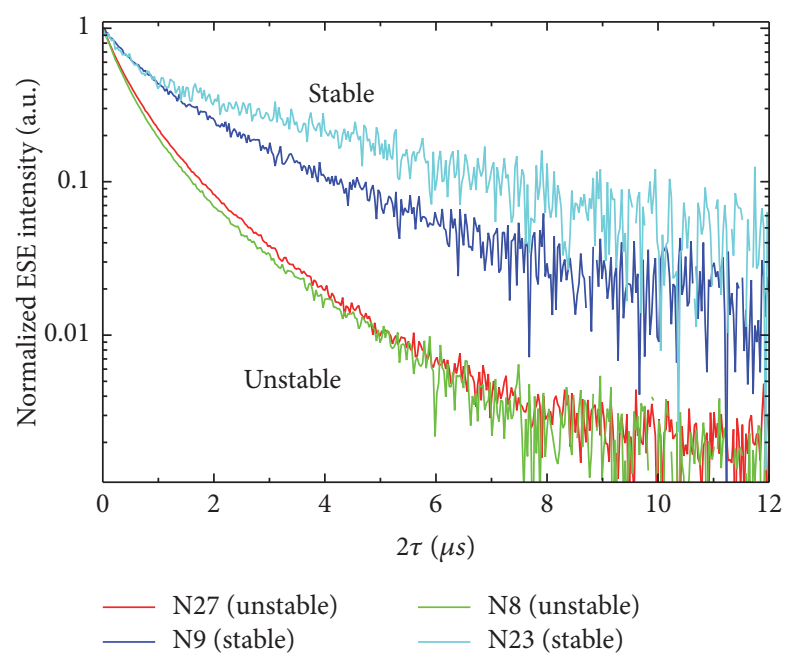

FIGURE 3: The experimental kinetics (semi-log plot) of electron spin echo ESE decays in samples of stable (numbers 9 and 23, blue and light blue curves) and unstable (numbers 8 and 27, red and green curves) atherosclerotic plaques at $T=8 \mathrm{~K}$ and magnetic field $B_{\text {rel }}=$ $3360 \mathrm{mT}$.

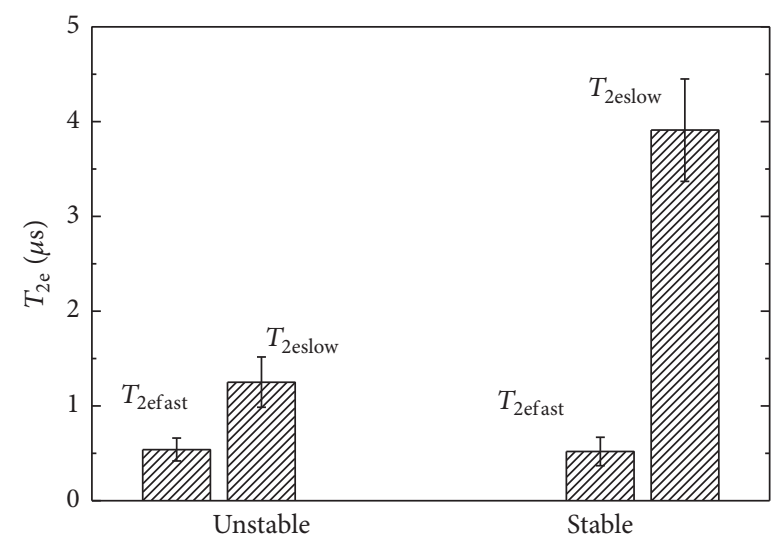

FIgURE 4: The mean values for fast $T_{2 \text { efast }}$ and slow $T_{\text {2eslow }}$ items of ESE decays with the corresponding SEM for $n=6$ (stable) and $n=$ 21 (unstable) plaques. The numeric values with the corresponding statistical analysis are given in Table 1.

"slow." As it can be seen (Figure 4, Table 1), while the fast relaxation component at the beginning of the ESE decay is practically the same for stable and unstable plaques, stable plaques have longer relaxation tales $\left(T_{2 \text { eslow }}\right.$ of about 3 times longer than for the unstable APs, $p<0.01)$.

\section{Discussion}

Let us firstly discus the $T_{2 \mathrm{e}}$ curves (Figures 3 and 4 ). Transverse/phase-memory electronic relaxation time $T_{2 \mathrm{e}}$ is one of the basic numerical values derived from the pulsed experiments. In homogeneous systems with small amount of paramagnetic species, $T_{2 \mathrm{e}}$ is inversely proportional to the concentration of paramagnetic centers and very sensitive to the changes in their local surrounding $[17,28]$. Our data indicate that the concentration of $\mathrm{Mn}^{2+}$ ions in the unstable plaques are higher than in the stable APs at least at the local surrounding of $\mathrm{Mn}^{2+}$ ions. Putting it in another way, the manganese ions in stable plaques are distributed more homogeneously or/and have less total concentration.

In papers of Lozhkin et al. [29, 30], human aorta specimens obtained from autopsy of patients with atherosclerosis were investigated. A negative correlation between the concentration of manganese ions and degree of AP calcification was found. These findings are in a contradiction with the widely accepted findings in the recent past opinion that the more calcified AP generally is more stable.

The modern investigations show that the calcification is not directly and independently associated with plaque rupture [5, 31]. Nevertheless, to date one can state that, regardless of the nature of AP calcification, its assessment to predict complications for the cardiovascular system turned to be higher than the traditional approaches to risk analysis. We hope that our further research by using the presented and the more advanced EPR approaches like electron-nuclear double resonance (ENDOR, [17, 21]) would allow for finding either positive or negative correlation between the status of $\mathrm{AP}$ and $\mathrm{Mn}^{2+}$ environment in the conditions of the microand macrocalcifications.

Secondly, we discuss the obtained EPR spectra (Figure 2). As we have already mentioned, the gathered set of the experimental facts allows for ascribing the obtained spectra to $\mathrm{Mn}^{2+}$ ions in hydroxyapatite. The incorporation of small amount of $\mathrm{Mn}^{2+}$ ions into the hydroxyapatite structure does not lead to any substantial alterations in the parameters of the crystallographic unit cell and does not affect hydroxyapatite crystallinity $[32,33]$. Surprisingly, the linewidths of the EPR spectra components in APs are quite narrow to distinguish the different contributions (cf. with the EPR of $20 \mathrm{~nm}$ synthetical Mn-HAp in Figure 1). It is evidenced that the obtained spectra belong to $\mathrm{Mn}^{2+}$ ions embedded into the crystal phase of the organomineral matrix.

The recent theoretical research [33] shows that $\mathrm{Mn}$ substituted HAp is the more stable structure than other divalent metal (like $\mathrm{Fe}^{2+}$ or $\mathrm{Co}^{2+}$ ) substituted hydroxyapatites. It might be a reason why only manganese (and carboncentered FR) paramagnetic complexes but not $\mathrm{Cu}^{2+}, \mathrm{Fe}^{2+}$, or $\mathrm{Fe}^{3+}$ containing ones, for example, are revealed in our measurements.

A location (position) of the introduced manganese in either biogenic material or synthesized samples is still debated $[17,32,33]$. As for the natural apatites, two nonequivalent calcium positions $\mathrm{Ca}(1)$ and $\mathrm{Ca}(2)$ distinguishable in the HAp structure are proposed for the $\mathrm{Mn}^{2+}$ substitutions. The $\mathrm{Ca}(1)$ ions lie along a line parallel to the crystal $c$-axis and are connected to each other by three shared oxygen ions resulting in a " $\mathrm{Ca}(1)$ channel." The $\mathrm{Ca}(2)$ ions form perpendicular to the $c$-axis triangles with two hydroxyl groups. This scheme forms an "OH channel."

It is conventionally assumed that $\mathrm{Mn}^{2+}$ ions preferentially occupy the $\mathrm{Ca}(1)$ position in the crystallographic unit cell but can be also distributed between the $\mathrm{Ca}(1)$ and $\mathrm{Ca}(2)$ positions $[17,32,33]$. In principle, EPR techniques should feel 
the difference between these locations. But up to the present, there are still no reliable calculations of $\mathrm{Mn}^{2+}$ EPR parameters for these positions while the more or less convincing experiments are done only for the natural apatite crystals; that is, they are derived from the angular dependencies of their EPR spectra (see [17] and references therein). This experimental approach, obviously, is not applicable for the AP calcium deposits. One of the obstacles that hinder the application of nuclear magnetic resonance (NMR) is less with respect to EPR sensitivity for investigations of small amounts of the clinical materials. Thus, to date we can assume that for the stable plaque a scheme for which $\mathrm{Mn}^{2+}$ substitutes mainly one calcium position (let us say $\mathrm{Ca}(1)$ ) is realized while in the unstable plaque both positions $\mathrm{Ca}(1)$ and $\mathrm{Ca}(2)$ are filled. This conclusion correlates with the differences in the $T_{2 \mathrm{e}}$ curves; for stable plaques, $\mathrm{Mn}^{2+}$ is distributed more homogeneously than for the unstable that leads to the slower relaxation times $T_{\text {2eslow }}$ for the stable plaques.

Thirdly, we discuss the possible origin of the detected manganese complexes. Lozhkin et al. speculated that the decrease in the manganese content with the increasing of AP calcification could be connected with the deficiency of mitochondrial Mn-SOD [29, 30]. However, now this assumption does not seem to be quite reasonable, because it is apparently not all of $\mathrm{Mn}^{2+}$ in AP has intracellular localization being in the mitochondrial Mn-SOD.

It is very likely that this pool of $\mathrm{Mn}^{2+}$ is formed as a result of the high-rate disintegration of the AP cells during atherogenesis. This pool of extracellular $\mathrm{Mn}^{2+}$, available in structures of the tissue matrix, could grow with the degree of the instability of AP which is accompanied by active cell death and disintegration. It is known that the apoptosis of resident macrophages is one of the earliest pathogenic events during atherosclerotic lesion of the vessel wall [34]. On the other hand, an increase of Mn-SOD expression in cells prevents the development of atherosclerosis and as might be expected the formation of unstable AP [1]. Mn-SOD activity increases significantly in the intima of an atherosclerotic artery [35]. Essential factor affecting activity of the enzyme is oxidized low-density lipoprotein (oxLDL), which have a damaging effect on the macrophages and plays a key role in the development of atherosclerosis.

\section{Conclusion}

For the first time, to the best of our knowledge, the pulsed Wband EPR comparative studies of a series of atherosclerotic plaques obtained during carotid endarterectomy in patients with atherosclerosis are presented. Due to the high sensitivity and small amount of tissues required for the W-band EPR, the samples of less than $1 \mathrm{~mm}$ in cross section could be investigated. In all of the obtained species, EPR spectra due to the presence of $\mathrm{Mn}^{2+}$ ions in a crystal matrix are observed. The spectral parameters of $\mathrm{Mn}^{2+}$ are shown to be different and ascribed to two possible locations in the hydroxyapatite constituent of the atherosclerotic plaques. The obtained difference in the electronic transverse relaxation $T_{2 \mathrm{e}}$ can indicate the higher local concentration of $\mathrm{Mn}^{2+}$ ions in the unstable plaques, that is, the more homogeneous distribution of $\mathrm{Mn}^{2+}$ ions in stable plaques. Therefore, it seems to be that stable and unstable AP differ in the molecular organization of their hydroxyapatite components. Simultaneously, we have confirmed the fact that the amount of calcium deposits is not connected with the AP instability, at least directly.

We realize that pulsed and high-field EPR due to the high equipment price and need of liquid helium probably would not be used for rapid determination of AP stability in the near future. But, as we showed, the detection and study of the spectral and relaxation characteristics of the intrinsic paramagnetic centers can be fruitfully used as a very sensitive tool for gathering additional information (which is inaccessible to other methods and techniques) about the content and morphology of the investigated tissues.

\section{Competing Interests}

The authors declare that there is no conflict of interests regarding the publication of this paper.

\section{Acknowledgments}

The work is devoted to Dr. Igor N. Kurkin on occasion if his 75th birthday. The authors also thank Airat Kiiamov for XRD measurements and Timur Biktagirov for helpful discussions. The work was done in the framework of the program of competitive growth of Kazan Federal University and funded by the subsidy for the state assignment in the sphere of scientific activity.

\section{References}

[1] F. Sharifpanah and H. Sauer, "Reactive oxygen species, oxidative stress, and cardiovascular diseases," in Oxidative Stress and Antioxidant Protection: The Science of Free Radical Biology and Disease, D. Armstrong and R. D. Stratton, Eds., chapter 17, pp. 281-307, John Wiley \& Sons, Hoboken, NJ, USA, 1st edition, 2016.

[2] R. G. González, J. A. Hirsch, M. H. Lev, P. W. Schaefer, and L. H. Schwamm, Eds., Acute Ischemic Stroke Imaging and Intervention, Springer, Berlin, Germany, 2011.

[3] G. Maiolino, G. Rossitto, P. Caielli, V. Bisogni, G. P. Rossi, and L. A. Calò, "The role of oxidized low-density lipoproteins in atherosclerosis: the myths and the facts," Mediators of Inflammation, vol. 2013, Article ID 714653, 13 pages, 2013.

[4] E. Aikawa, Ed., Cardiovascular Imaging Arterial and Aortic Valve Inflammation and Calcification, Springer International Publishing, 2015.

[5] J. D. Hutcheson, C. Goettsch, S. Bertazzo et al., "Genesis and growth of extracellular-vesicle-derived microcalcification in atherosclerotic plaques," Nature Materials, vol. 15, no. 3, pp. 335343, 2016.

[6] V. A. Abdul'yanov, L. F. Galiullina, A. S. Galyavich et al., "Stationary and high-frequency pulsed electron paramagnetic resonance of a calcified atherosclerotic plaque," Journal of Experimental and Theoretical Physics Letters, vol. 88, no. 1, pp. 69-73, 2008.

[7] M. R. Gafurov, B. V. Yavkin, T. B. Biktagirov et al., "Atherosclerotic plaque and hydroxyapatite nanostructures studied by 
high-frequency EPR," Magnetic Resonance in Solids, vol. 15, no. 1, article 13102, 2013.

[8] D. Bazin, M. Daudon, C. Combes, and C. Rey, "Characterization and some physicochemical aspects of pathological microcalcifications," Chemical Reviews, vol. 112, no. 10, pp. 5092-5120, 2012.

[9] S. V. Dorozhkin, "Calcium orthophosphates (CaPO4): occurrence and properties," Progress in Biomaterials, vol. 5, no. 1, pp. 9-70, 2016.

[10] M. Šupová, "Substituted hydroxyapatites for biomedical applications: a review, Ceramics International, vol. 41, no. 8, pp. 9203-9231, 2015.

[11] J. Kolmas, E. Groszyk, and D. Kwiatkowska-Rózycka, "Substituted hydroxyapatites with antibacterial properties," BioMed Research International, vol. 2014, Article ID 178123, 15 pages, 2014.

[12] A. P. Burlaka, I. I. Ganusevich, M. R. Gafurov, S. M. Lukin, and E. P. Sidorik, "Stomach cancer: interconnection between the redox state, activity of MMP-2, MMP-9 and stage of tumor growth," Cancer Microenvironment, vol. 9, no. 1, pp. 27-32, 2016.

[13] A. P. Burlaka, M. R. Gafurov, K. B. Iskhakova et al., "Electron paramagnetic resonance in the experimental oncology: implementation examples of the conventional approaches," BioNanoScience, vol. 6, no. 4, pp. 431-436, 2016.

[14] S. L. O’Neal and W. Zheng, "Manganese toxicity upon overexposure: a decade in review," Current Environmental Health Reports, vol. 2, no. 3, pp. 315-328, 2015.

[15] C. Li and H.-M. Zhou, "The role of manganese superoxide dismutase in inflammation defense," Enzyme Research, vol. 2011, no. 1, Article ID 387176, 6 pages, 2011.

[16] R. Stocker and J. F. Keaney Jr., "Role of oxidative modifications in atherosclerosis," Physiological Reviews, vol. 84, no. 4, pp. 13811478, 2004.

[17] M. Gafurov, T. Biktagirov, G. Mamin et al., “The Interplay of manganese and nitrate in hydroxyapatite nanoparticles as revealed by pulsed EPR and DFT," Physical Chemistry Chemical Physics, vol. 17, no. 31, pp. 20331-20337, 2015.

[18] M. Gafurov, T. Biktagirov, B. Yavkin et al., "Nitrogencontaining species in the structure of the synthesized nanohydroxyapatite," JETP Letters, vol. 99, no. 4, pp. 196-203, 2014.

[19] T. B. Biktagirov, M. R. Gafurov, G. V. Mamin et al., "A study of hydroxyapatite nanocrystals by the multifrequency EPR and ENDOR spectroscopy methods," Optics and Spectroscopy, vol. 116, no. 5, pp. 715-720, 2014.

[20] T. Biktagirov, M. Gafurov, G. Mamin, E. Klimashina, V. Putlayev, and S. Orlinskii, "Combination of EPR measurements and DFT calculations to study nitrate impurities in the carbonated nanohydroxyapatite," The Journal of Physical Chemistry A, vol. 118, no. 8, pp. 1519-1526, 2014.

[21] B. V. Yavkin, G. V. Mamin, S. B. Orlinskii et al., " $\mathrm{Pb}^{3+}$ radiation defects in $\mathrm{Ca}_{9} \mathrm{~Pb}\left(\mathrm{PO}_{4}\right)_{6}(\mathrm{OH})_{2}$ hydroxyapatite nanoparticles studied by high-field (W-band) EPR and ENDOR," Physical Chemistry Chemical Physics, vol. 14, no. 7, pp. 2246-2249, 2012.

[22] "Electron paramagnetic resonance investigations of biological systems by using spin labels, spin probes, and intrinsic metal ions, part A," in Methods in Enzymology, P. Z. Qin and K. Warncke, Eds., vol. 563, pp. 2-684, Elsevier, 2015.

[23] "Electron paramagnetic resonance investigations of biological systems by using spin labels, spin probes, and intrinsic metal ions, Part B," in Methods in Enzymology, P. Z. Qin and K. Warncke, Eds., vol. 564, pp. 603-613, Elsevier, Amsterdam, Netherlands, 2015.
[24] A. C. Gray-Weale, J. C. Graham, J. R. Burnett, K. Byrne, and R. J. Lusby, "Carotid artery atheroma: comparison of preoperative B-mode ultrasound appearance with carotid endarterectomy specimen pathology," Journal of Cardiovascular Surgery, vol. 29, no. 6, pp. 676-681, 1988.

[25] G. Geroulakos, G. Ramaswami, A. Nicolaides et al., "Characterization of symptomatic and asymptomatic carotid plaques using high-resolution real-time ultrasonography," British Journal of Surgery, vol. 80, no. 10, pp. 1274-1277, 1993.

[26] S. Gražulis, A. Daškevič, A. Merkys et al., "Crystallography Open Database (COD): An open-access collection of crystal structures and platform for world-wide collaboration," Nucleic Acids Research, vol. 40, no. D1, pp. D420-D427, 2012.

[27] J. Pelisek, G. Well, C. Reeps et al., "Neovascularization and angiogenic factors in advanced human carotid artery stenosis," Circulation Journal, vol. 76, no. 5, pp. 1274-1282, 2012.

[28] G. V. Mamin, M. R. Gafurov, R. V. Yusupov et al., “Toward the asphaltene structure by electron paramagnetic resonance relaxation studies at high fields (3.4 T)," Energy \& Fuels, vol. 30, no. 9, pp. 6942-6946, 2016.

[29] A. P. Lozhkin, T. B. Biktagirov, V. A. Abdul'yanov et al., "Manganese as a potential marker of atherogenesis," Doklady Biochemistry and Biophysics, vol. 434, no. 1, pp. 254-256, 2010.

[30] A. P. Lozhkin, T. B. Biktagirov, V. A. Abdul'yanov et al., "Manganese in atherogenesis: detection, origin, and a role," Biochemistry (Moscow) Supplement Series B: Biomedical Chemistry, vol. 5, no. 2, pp. 158-162, 2011.

[31] F. Otsuka, K. Sakakura, K. Yahagi, M. Joner, and R. Virmani, "Has our understanding of calcification in human coronary atherosclerosis progressed?" Arteriosclerosis, Thrombosis, and Vascular Biology, vol. 34, no. 4, pp. 724-736, 2014.

[32] J. Kolmas, M. Jabłoński, A. Ślósarczyk, W. Kolodziejski, and W. Wei, "Solid-state NMR study of $\mathrm{Mn}^{2+}$ for $\mathrm{Ca}^{2+}$ substitution in thermally processed hydroxyapatites," Journal of the American Ceramic Society, vol. 98, no. 4, pp. 1265-1274, 2015.

[33] M. E. Zilm, L. Chen, V. Sharma et al., "Hydroxyapatite substituted by transition metals: experiment and theory," Phys. Chem. Chem. Phys., vol. 18, no. 24, pp. 16457-16465, 2016.

[34] K. J. Moore, F. J. Sheedy, and E. A. Fisher, "Macrophages in atherosclerosis: a dynamic balance," Nature Reviews Immunology, vol. 13, no. 10, pp. 709-721, 2013.

[35] R. Kinscherf, H.-P. Deigner, C. Usinger et al., "Induction of mitochondrial manganese superoxide dismutase in macrophages by oxidized LDL: its relevance in atherosclerosis of humans and heritable hyperlipidemic rabbits," FASEB Journal, vol. 11, no. 14, pp. 1317-1328, 1997. 


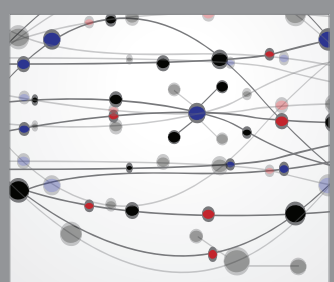

The Scientific World Journal
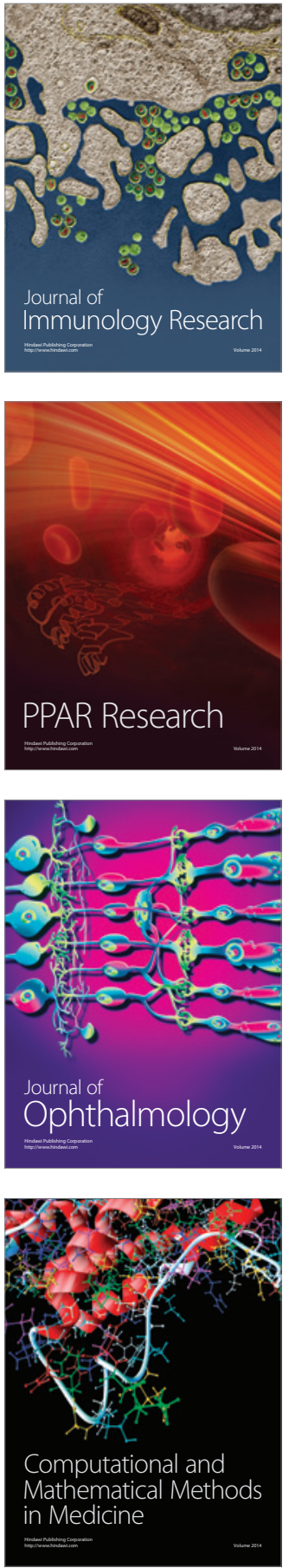

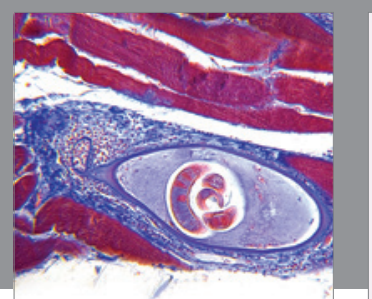

Gastroenterology Research and Practice

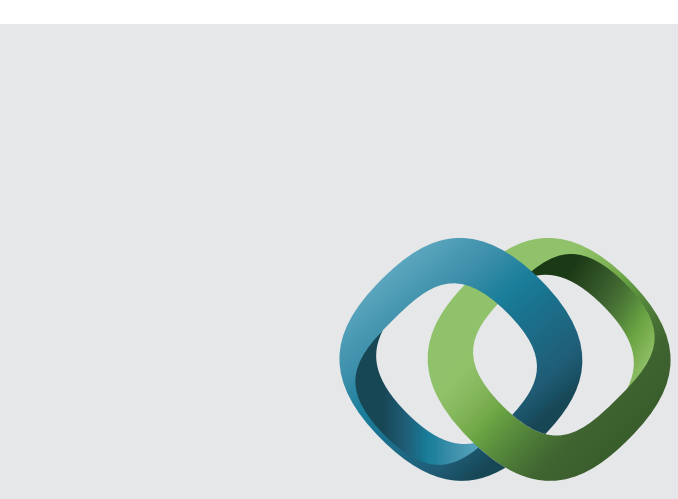

\section{Hindawi}

Submit your manuscripts at

http://www.hindawi.com
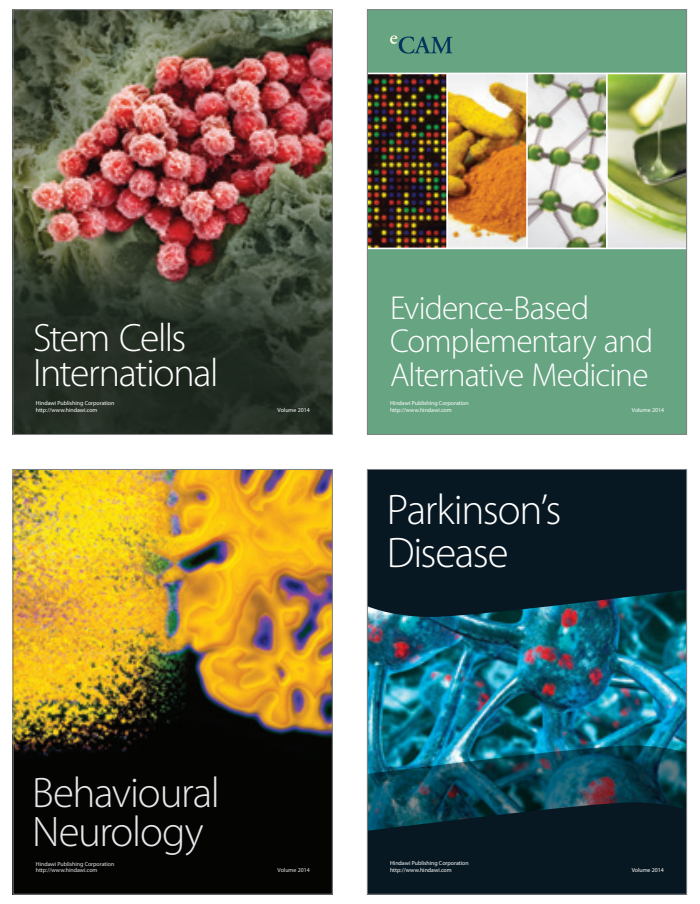
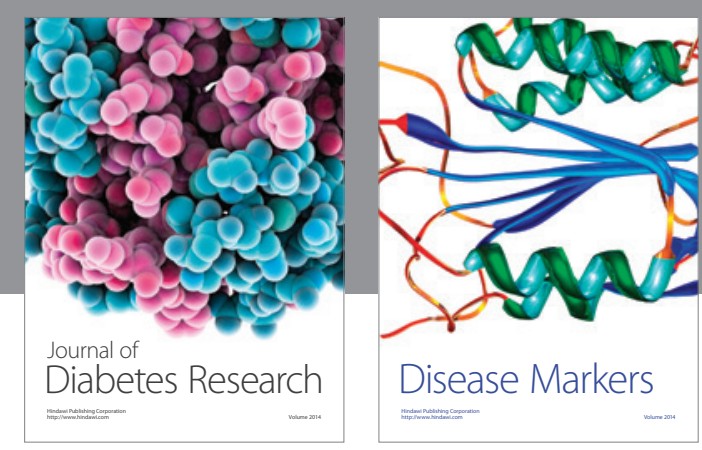

Disease Markers
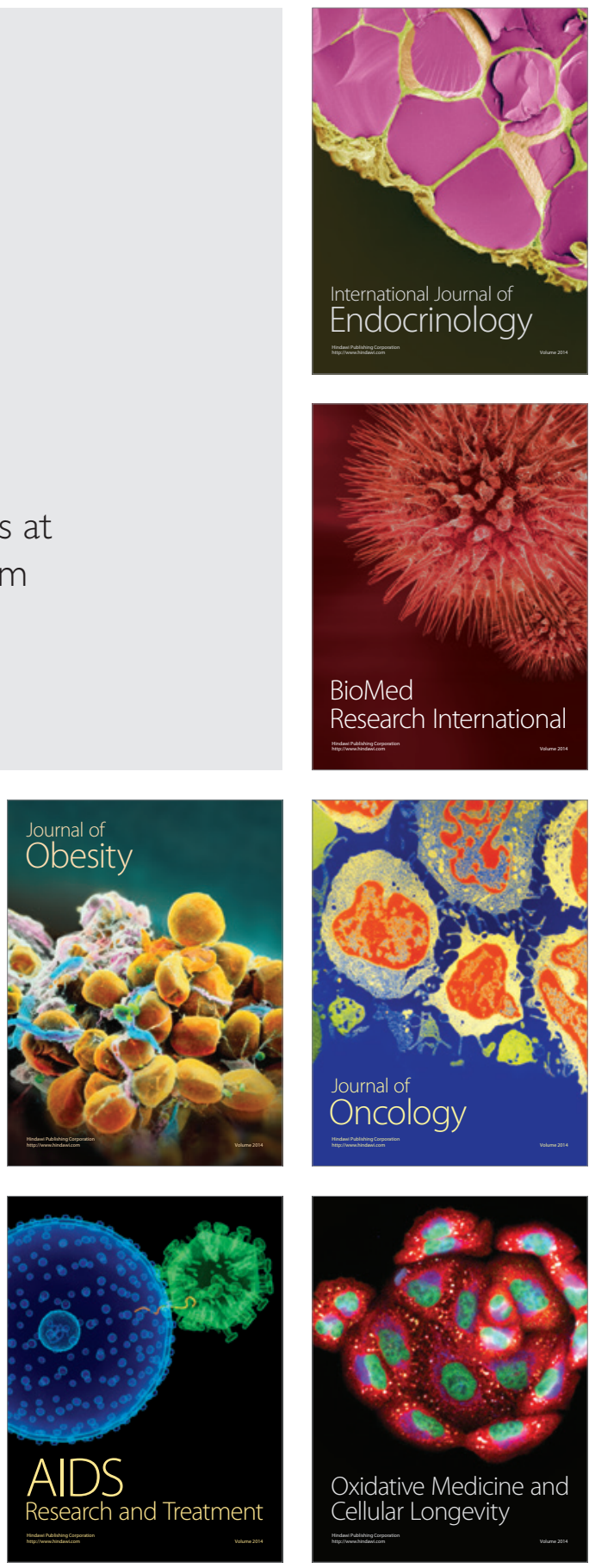